\title{
Vpu serine 52 dependent counteraction of tetherin is required for HIV-1 replication in macrophages, but not in ex vivo human lymphoid tissue
}

Michael Schindler ${ }^{1 *}$, Devi Rajann ${ }^{2,3}$, Carina Banning ${ }^{1}$, Peter Wimmer ${ }^{1}$, Herwig Koppensteiner ${ }^{1}$, Alicja Iwanski ${ }^{1}$, Anke Specht ${ }^{2}$, Daniel Sauter ${ }^{2}$, Thomas Dobner ${ }^{1}$, Frank Kirchhoff ${ }^{2}$

\begin{abstract}
Background: The human immunodeficiency virus type 1 (HIV-1) Vpu protein degrades CD4 and counteracts a restriction factor termed tetherin (CD317; Bst-2) to enhance virion release. It has been suggested that both functions can be genetically separated by mutation of a serine residue at position 52. However, recent data suggest that the $\mathbf{S} 52$ phosphorylation site is also important for the ability of Vpu to counteract tetherin. To clarify this issue, we performed a comprehensive analysis of HIV-1 with a mutated casein kinase-II phosphorylation site in Vpu in various cell lines, primary blood lymphocytes (PBL), monocyte-derived macrophages (MDM) and ex vivo human lymphoid tissue (HLT).
\end{abstract}

Results: We show that mutation of serine 52 to alanine (S52A) entirely disrupts Vpu-mediated degradation of CD4 and strongly impairs its ability to antagonize tetherin. Furthermore, casein-kinase II inhibitors blocked the ability of Vpu to degrade tetherin. Overall, Vpu S52A could only overcome low levels of tetherin, and its activity decreased in a manner dependent on the amount of transiently or endogenously expressed tetherin. As a consequence, the S52A Vpu mutant virus was unable to replicate in macrophages, which express high levels of this restriction factor. In contrast, HIV-1 Vpu S52A caused CD4+ T-cell depletion and spread efficiently in ex vivo human lymphoid tissue and PBL, most likely because these cells express comparably low levels of tetherin.

Conclusion: Our data explain why the effect of the S52A mutation in Vpu on virus release is cell-type dependent and suggest that a reduced ability of Vpu to counteract tetherin impairs HIV-1 replication in macrophages, but not in tissue CD4+ T cells.

\section{Background}

$\mathrm{Vpu}$ is an accessory HIV-1 protein of 16-kDa expressed late during the viral life cycle [1], and it is known to perform two major functions. Firstly, Vpu targets CD4 for degradation in the endoplasmic reticulum [2-4]. Secondly, it promotes virion release in a cell-type dependent manner by counteracting a host restriction factor that can be induced by interferon-alpha [5]. This factor has been identified as CD317/BST-2 and is termed tetherin, because it "tethers" nascent virions to cell

\footnotetext{
* Correspondence: michael.schindler@hpi.uni-hamburg.de

'Heinrich-Pette-Institute for Experimental Virology and Immunology, Martinistrasse 52, 20251 Hamburg, Germany
}

\section{Martinistrasse 52, 20251 Hamburg, Germany}

membranes [6,7]. From a mechanistic point of view Vpu binds to $\mathrm{CD} 4$, is phosphorylated at two serine residues at positions 52 and 56 by casein kinase II (CK-II), and recruits the E3-ubiquitin ligase substrate recognition factor $\beta$-TrCP. Subsequently, CD4 is ubiquitinated and degraded by the cellular proteasome $[1,4,8]$. Recent studies suggest that $\mathrm{Vpu}$ may induce internalization and degradation of tetherin by the same pathway [9-11]. In contrast, earlier work suggested that phosphorylation of S52 and S56 in the cytosolic domain of Vpu by CK-II is critical for $\mathrm{CD} 4$ degradation, but not for the enhancement of virion release $[8,12-15]$. Since the enhancing effect of $\mathrm{Vpu}$ on HIV-1 release is cell type dependent $[5,16,17]$, some of these seeming discrepancies may

(c) 2010 Schindler et al; licensee BioMed Central Ltd. This is an Open Access article distributed under the terms of the Creative Commons Attribution License (http://creativecommons.org/licenses/by/2.0), which permits unrestricted use, distribution, and 
result from different levels of tetherin expression and hence a differential requirement for effective tetherin antagonism.

In the present study, we performed a comprehensive analysis of Vpu function in HIV-1 infected primary cells and ex vivo tissue. In comparison to wildtype $\mathrm{Vpu}$, the S52A mutant was strongly impaired in its ability to counteract tetherin, permitting viral release only at low levels of tetherin expression. These results may explain why HIV-1 encoding S52A Vpu caused CD4+ T-cell depletion and replicated with wildtype-like efficiency in lymphoid cells and HLT ex vivo, but not in macrophages that express higher levels of tetherin. In sum, our data suggest that the ability of $\mathrm{Vpu}$ to counteract tetherin is an important determinant for HIV-1 cell tropism.

\section{Results \\ Vpu S52A impairs tetherin and CD4 degradation in transfected 293T cells}

For functional analyses, we generated untagged and AU1-tagged forms of the wildtype and S52A HIV-1 NL4-3 Vpus and verified their expression by Western blot analysis (Fig. 1A). Down-modulation of CD4 from the cell surface was measured by flow cytometric analysis of Jurkat $\mathrm{T}$ cells transiently transfected with vectors co-expressing Vpu and GFP via an internal ribosomal entry site (IRES). Transport of CD4 to the cell surface was measured by co-transfection of 293T cells with CD4 and constructs expressing GFP alone or together with Vpu. Wildtype Vpu caused about 2-fold reduced levels of CD4 expression on Jurkat T cells and efficiently blocked the transport of newly synthesized CD4 to the surface of 293T cells (Fig. 1B, C). In contrast, the S52A Vpu was inactive in both assays (Fig. 1B, C).

It has been shown that Vpu reduces the total levels of cellular tetherin, and it has been suggested that this effect may be important for its capability to promote virus release $[9,10,18,19]$. To test whether the S52A change affects tetherin degradation by $\mathrm{Vpu}$, we generated an N-terminally eCFP-tagged version of tetherin. Confocal microscopy showed that the fusion protein had a subcellular localization comparable to endogenous tetherin and inhibited viral particle release (data not shown). Degradation of total cellular tetherin was measured by co-transfection of eCFP-tetherin with the various $\mathrm{Vpu} / \mathrm{GFP}$ constructs. Expression of wildtype Vpu resulted in about $50 \%$ reduction in the number of tetherin expressing cells, whereas the S52A Vpu degraded tetherin in only about $20 \%$ of cells (Fig. 1D). It has been shown that $\mathrm{Vpu}$ is phosphorylated by CK-II [12], but the importance of an active CK-II for the ability of $\mathrm{Vpu}$ to degrade tetherin is not known. Therefore, we measured Vpu-mediated tetherin degradation in the presence of different CK-II inhibitors (Fig. 1E). Tyrphostin inhibited degradation of tetherin by $\mathrm{Vpu}$ already at $25 \mu \mathrm{M}$ whereas Cay10577 and DRB did so in a dosedependent manner, demonstrating the importance of CK-II activity for the degrading effects of $\mathrm{Vpu}$ on tetherin (Fig. 1E). These results show that mutation of S52A is sufficient to entirely disrupt the effect of $\mathrm{Vpu}$ on CD4 and establish at a single cell level that an intact CK-II phosphorylation site as well as active CK-II are important for degradation of tetherin by $\mathrm{Vpu}$.

\section{The S52A Vpu is only able to antagonize tetherin at low} expression levels

Vpu S52A still degraded tetherin to some extent in cells co-transfected with $\mathrm{Vpu}$ and tetherin expression plasmids (Figures 1D and 1E). Therefore, we speculated that Vpu S52A might be able to enhance HIV-1 release at low levels of tetherin expression. We co-transfected 293 T cells with WT, Vpu-defective, and Vpu S52A expressing proviral constructs and different amounts of tetherin ranging from $100 \mathrm{ng}$ (1:50; ratio transfected tetherin:provirus) to $10 \mathrm{ng}$ (1:500); and we measured cellular as well as released p24 by a quantitative Western blot two days later (Fig. 2A and 2B). As expected, 293T cells expressing very low $10 \mathrm{ng}$ (1:500) levels of tetherin released p24 independently of functional $\mathrm{Vpu}$ expression. However, transfection of $20 \mathrm{ng}$ (1:250) tetherin already reduced virus release of $\mathrm{Vpu}$-defective HIV-1 by about $50 \%$. At these levels of tetherin expression the S52A Vpu enhanced p24 release as efficiently as the wildtype $\mathrm{Vpu}$ protein. In contrast, virus release of the mutant was suppressed by more than one order of magnitude at higher levels of tetherin expression (Fig. $2 \mathrm{~A}, \mathrm{~B})$. Of note, we did not detect any p24 in the supernatant of cells expressing Vpu-defective HIV-1 when tetherin was transfected at a ratio of 1:50. As a control, we measured virion content by ELISA in supernatants of transfected cells before the virus was pelleted. These analyses demonstrated that results obtained by ELISA correlated highly significantly $(R=0.9159 ; \mathrm{p}<0.0001)$ with the quantitative WB results (Additional file 1). In sum, the S52A change severely attenuates the ability of $\mathrm{Vpu}$ to enhance HIV-1 release with increasing levels of tetherin expression.

Previously, it was reported that macrophages and primary T-cells, the main HIV-1 target cells in vivo, express different amounts of endogenous tetherin [20]. Prompted by our results, we speculated that Vpu with a mutated CK-II site might not be able to counteract high levels of tetherin expression found in macrophages, but may replicate efficiently in T-cells that express low levels of tetherin. Since it is known that macrophages exert phenotypically high donor variations, we first aimed to investigate the levels of endogenous tetherin in macrophages from various donors in comparison to 


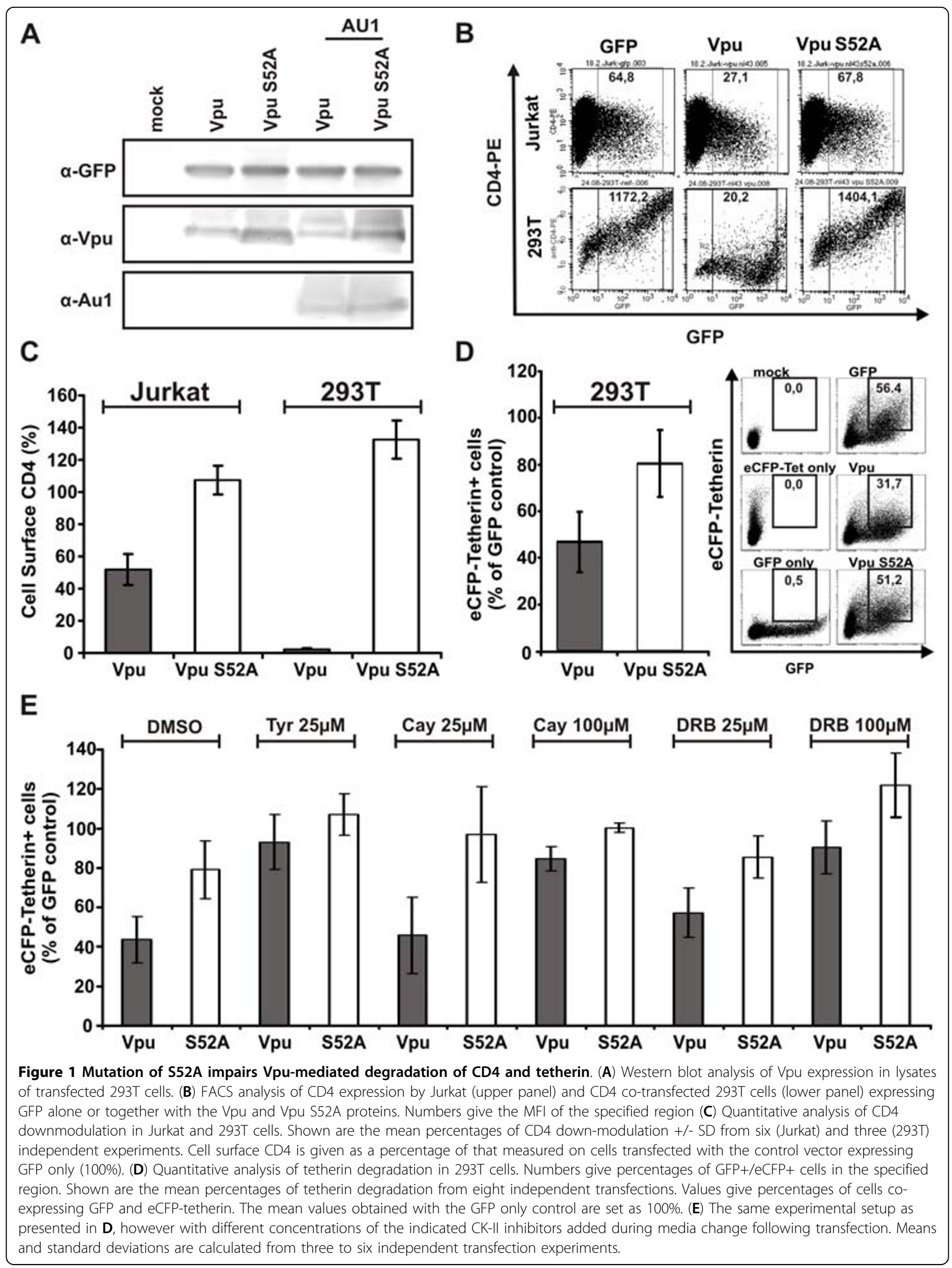






autologous T-cells (Fig. 2C). Western blot analysis revealed multiple bands, which is in agreement with previous findings showing that tetherin is glycosylated and can multimerize $[10,18,20]$. Untransfected $293 \mathrm{~T}$ cells that allow efficient release of HIV-1 particles in the presence and absence of $\mathrm{Vpu}$ did not express detectable levels of tetherin (Fig. 2B). Of note, macrophages expressed markedly higher levels of tetherin than PHAstimulated or unstimulated PBL (Fig. 2C). Thus, Vpu S52A might be differentially active in the enhancement of particle release from primary $\mathrm{T}$-cells and monocyte- derived macrophages (MDM) because it is only able to counteract tetherin at low expression levels.

Vpu S52A promotes virus release from HeLa-derived cells To investigate the effect of the S52A mutation in Vpu on HIV-1 release we constructed CXCR4(X4)- and CCR5 (R5)-tropic HIV-1 NL4-3 mutants carrying this change alone or in combination with a disrupted nef gene. The latter constructs were generated because Nef is known to down-modulate CD4 and to enhance viral infectivity and replication and may thus bias possible effects of the S52A change in Vpu [21-23]. Western blot analyses confirmed 
that all proviral constructs showed the expected differences in Vpu and Nef expression (Fig. 3A). Next, we decided to assess first the release of the different HIV-1 NL4-3 variants in the well established HeLa-derived P4CCR5 cells [24,25]. We transfected them with normalized quantities of proviral DNA and measured p24 content in the cell culture supernatant. Importantly, transfection efficiencies were comparable, since similar levels of Tatdependent expression of the LTR-driven $\beta$-galactosidase gene were detected in all cell lysates (data not shown). In agreement with the previous finding that $\mathrm{Vpu}$ is required for effective virus release from HeLa-derived cell lines
[16], the expression of wildtype Vpu resulted in about 5to 6-fold increased levels of p24 antigen in the culture supernatant. The S52A Vpu enhanced the release of progeny virions with similar efficiency, whereas Nef had no significant effect (Fig. 3B). This result was in line with our hypothesis that Vpu S52A can overcome relatively low levels of tetherin expression, because our P4-CCR5 cells expressed tetherin in a range comparably to unstimulated PBMCs (Additional file 2). Infection of P4-CCR5 cells with virus stocks containing normalized amounts of p24 (1 ng p24) [25] showed that only changes in nef, but not in $v p u$, impaired viral infectivity (Fig. 3C). Most
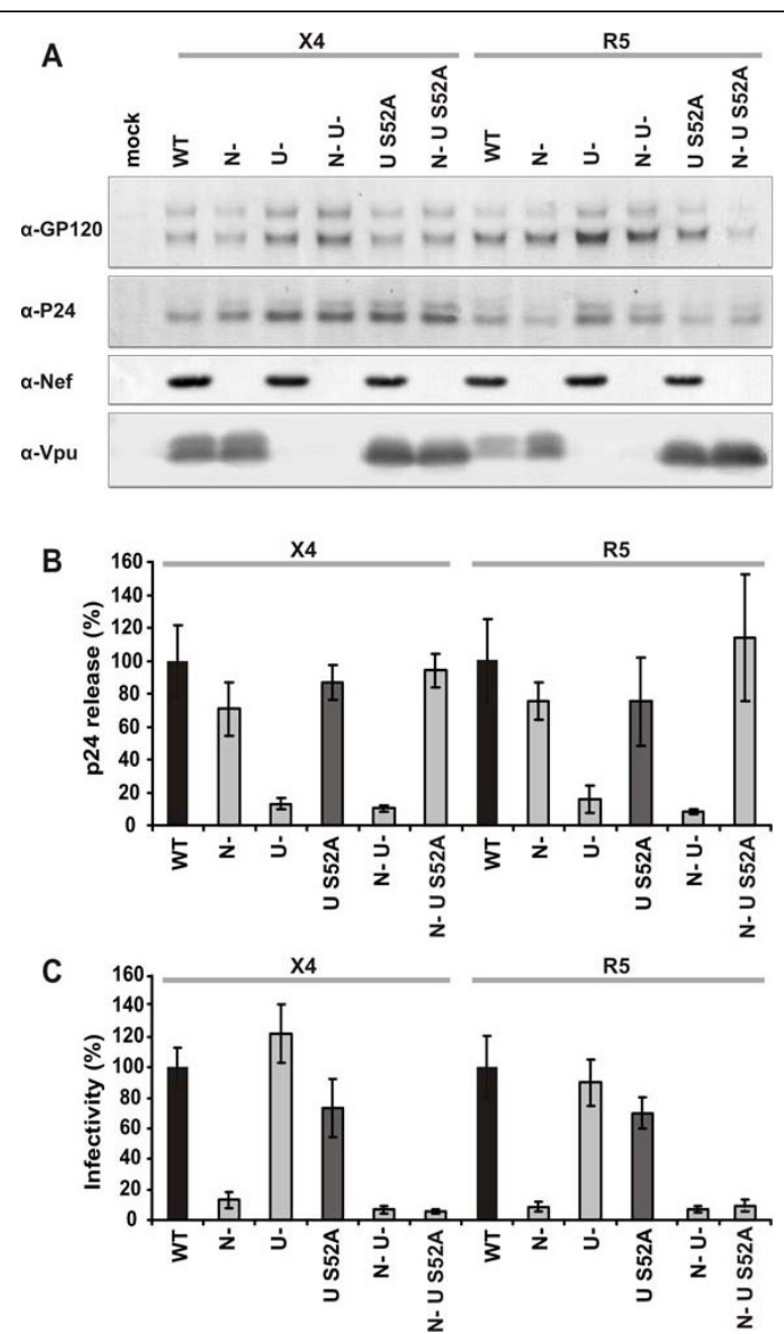

Figure 3 Vpu S52A does not impair HIV-1 release from P4-CCR5 cells. (A) Western blot analysis of viral gene expression in lysates of transfected 293T cells. (B) Viral particle release by P4-CCR5 cells transfected with the indicated X4 and R5 HIV-1 NL4-3 proviral constructs. P4CCR5 cells were transfected with $0.1 \mu \mathrm{g}$ proviral DNA in sextuplicates and p24 in the culture supernatants was quantified by p24 ELISA three days later. Measurement of the $\beta$-Gal activities in the cell lysates verified similar transfection efficiencies (not shown). Values give averages $+/-$ SD from two independent experiments with sextuplicate transfections and represent percentages compared to NL4-3 wildtype transfected cells (100\%). (C) P4-CCR5 indicator cells were infected in triplicate with virus stocks containing $1 \mathrm{ng}$ p24 antigen derived from 293T cells transfected with the indicated proviral constructs and $\beta$-Gal activity was determined three days later. Shown are average values $+/-$ SD from two independent experiments with triplicate infections of two independent virus stocks. Infectivity is given as percentage compared to infectivity of NL4-3 wildtype infected cells (100\%). Abbreviations, N-, Nef-defective; U-, Vpu-defective; S52A, VpuS52A. 
importantly, these findings demonstrated that the S52A $\mathrm{Vpu}$ is capable of enhancing virion release from HeLa derived P4-CCR5 cells that express relatively low levels of tetherin.

The S52A mutation in Vpu does not impair HIV-1 replication and cytopathicity in lymphoid tissue ex vivo It has been demonstrated that $\mathrm{Vpu}$ is critical for efficient HIV-1 replication and CD4+ T-cell depletion in HLT ex vivo $[26,27]$. This system allows productive HIV-1 infection without exogenous stimulation and mimics infection of lymphatic tissues, one of the major sites of viral replication in vivo [28]. To study the effect of the S52A change in Vpu on HIV-1 replication and cytopathicity, we infected HLT ex vivo with the X4 and R5 NL4-3 variants (Fig. 3A). Representative examples of replication results are presented in Figure 4A. Overall, we found that a defective $v p u$ gene reduced the production of wildtype X4 NL43 by $60 \%$ and of the R5-tropic derivative by $75 \%$ (Fig. 4B). Similarly, deletion of nef reduced cumulative virus production by about $75 \%$ (Fig. 4B). In contrast, the HIV-1 S52A Vpu mutation did not significantly attenuate HIV-1 replication (Fig. 4A and 4B). Consistent with the results of
A

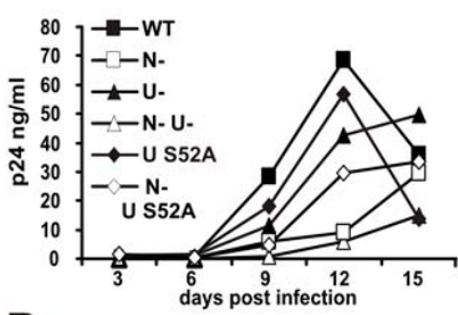

B
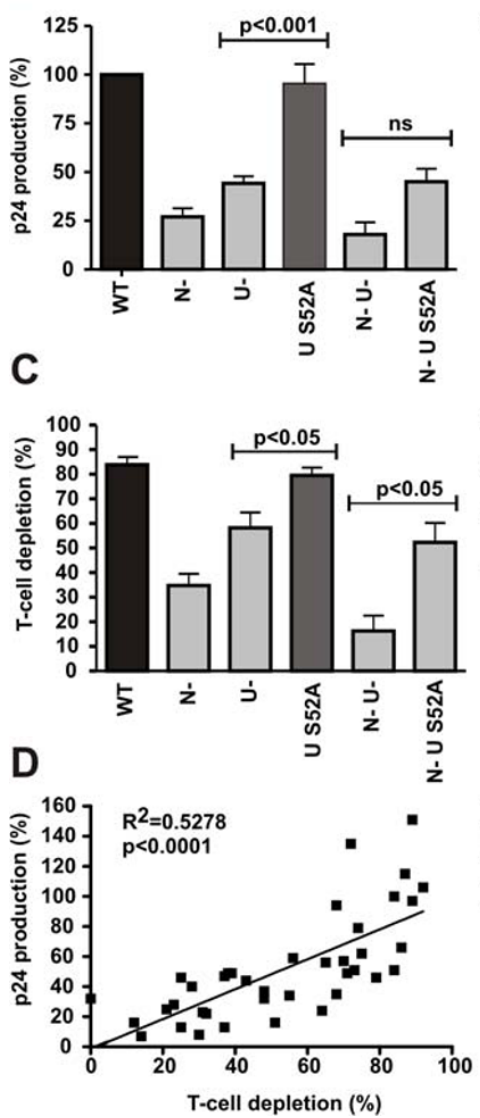

R5
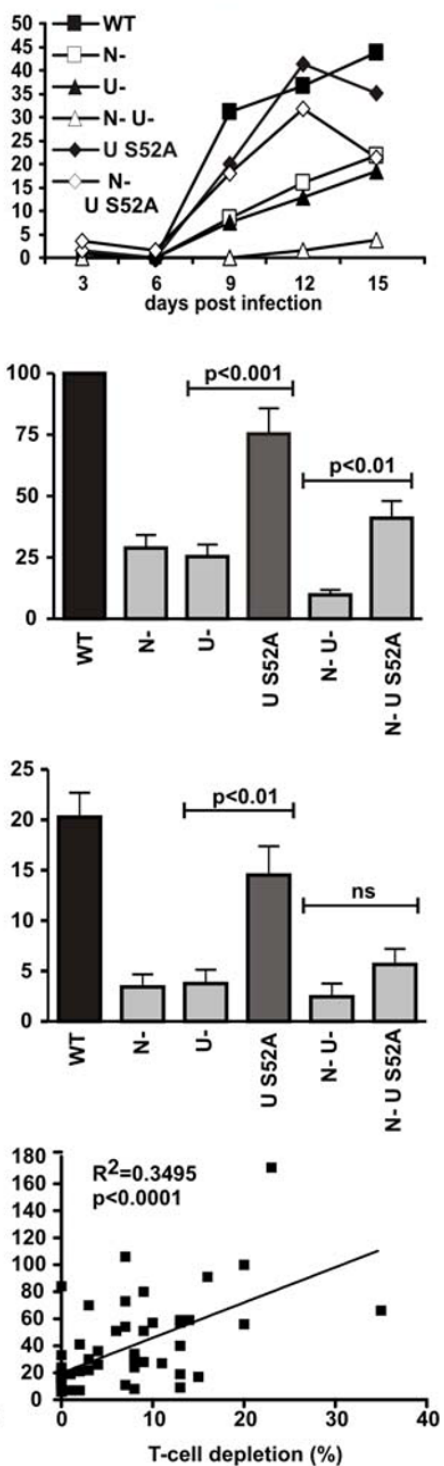

Figure 4 Vpu S52A is dispensable for HIV-1 replication and cytopathicity in ex vivo infected HLT. Representative replication kinetics (A) of the indicated X4 and R5 HIV-1 NL4-3 constructs. (B) Cumulative p24 production over 15 days and (C) CD4+ T cells depletion at the end of culture in tissues from eight (X4) and ten (R5) donors infected with the indicated HIV-1 variants. Values are given as percentages compared to cultures infected with NL4-3 wildtype (100\%). Shown are means +/- SEM. (D) Correlation between p24 production and CD4+ T-cell depletion. 
previous studies [26,27], wildtype X4 NL4-3 virus depleted the ex vivo infected tissues of $80 \%$ of X4-expressing CD4+ $\mathrm{T}$ cells, whereas the R5 HIV-1 derivative depleted $20 \%$ of R5+/CD4+ cells (Fig. 4C). Individual or combined deletions in $n e f$ and $v p u$ significantly reduced CD4+ T-cell depletion irrespectively of the viral coreceptor tropism, whereas the S52A mutation in Vpu had no significant effect (Fig. 4C). The efficiency of viral replication correlated well with CD4+ T-cell depletion (Fig. 4D) suggesting that these differences in cytopathicity resulted from lower numbers of infected cells, rather than from direct effects of Nef or Vpu on cell killing. These data show for the first time that the CK-II phosphorylation site in Vpu is not critical for effective viral spread and CD4+ T-cell depletion in ex vivo infected lymphoid tissue.

It has previously been established that HIV-1 replication in HLT occurs mainly in both activated and non-activated CD4+ T-cells [29] that express relatively low levels of tetherin (Fig. 2C, Additional file 2). Therefore, the wildtype like phenotype of HIV-1 Vpu S52A in HLT might be due to low tetherin expression levels in the relevant HIV-1 tissue target cells. Since it is difficult to isolate a sufficient number of CD4+ T-cells from these tissues to directly assess endogenous tetherin levels, we decided to investigate if replication of the HIV-1 variants in PBL mimics the situation in HLT. As expected, HIV-1 Vpu S52A replicated as efficiently as WT HIV-1 in cultures of primary blood lymphocytes, whereas Vpu-defective HIV-1 showed attenuated and delayed replication kinetics (Additional file 3 fig. S3a). Furthermore, electroporation of Jurkat T-cells with the proviral constructs and increasing amounts of tetherin expression plasmids confirmed that in T-cells the ability of $\mathrm{Vpu}$ S52A to enhance HIV-1 release also decreases in a tetherin-expression dependent manner (Additional file 3 fig. S3B).

\section{The S52A change in Vpu impairs HIV-1 replication in} macrophages

Macrophages express markedly higher levels of tetherin than PHA-stimulated or unstimulated PBL (Fig. 2C,
Additional file 2). Thus, we finally wanted to challenge the hypothesis that Vpu S52A might be impaired in the enhancement of particle release from infected MDM, because it is not able to counteract high tetherin expression levels. Therefore, we investigated the replicative capacity of the different R5-tropic viruses (Fig. 3A) in MDMs. In agreement with previous reports [30-33], only the disruption of $v p u$ but not of nef severely attenuated HIV-1 replication (Fig. 5). Most remarkably, the S52A mutation in $\mathrm{Vpu}$ impaired the replicative capacity of HIV-1 in macrophages as severely as the complete lack of Vpu function. Thus, Vpu S52A might be impaired in the enhancement of particle release from infected MDM, because it is not able to counteract tetherin at high expression levels.

Modulation of cell surface expressed CD4 and tetherin in HIV-1 infected PBL and macrophages

Currently, it is not known whether Vpu modulates cell surface expression of tetherin in primary $\mathrm{T}$-cells and macrophages. To address this, we generated proviral HIV-1 constructs containing wildtype or mutated $v p u$ genes co-expressing Nef and eGFP via an IRES [25,34]. PBL and MDM were infected with VSV-G pseudotyped viruses and assessed for the modulation of cell surface CD4 and tetherin by FACS. In agreement with previous reports [21,22], we found that inactivation of Nef more severely reduced than $\mathrm{Vpu}$ the ability of $\mathrm{HIV}-1$ to remove CD4 from the surface of infected primary T-cells (Fig. 6A). Nevertheless, the fact that the combined deletions had the most disruptive effects on cell surface CD4 expression demonstrated that both Nef as well as $\mathrm{Vpu}$ are important for effective removal of CD4. Moreover, the S52A change as well as inactivation of $\mathrm{Vpu}$ impaired the ability of HIV-1 to down-modulate CD4 to the same extent (Fig. 6A, left). Down-modulation of cell surface tetherin from HIV-1 infected PBL was clearly dependent on Vpu expression (Fig. 6A, right). Furthermore, the levels of cell surface tetherin in infected cells expressing S52A Vpu were significantly


Figure 5 Vpu S52A impairs HIV-1 replication in macrophages. Replication kinetics of wildtype NL4-3 and the indicated mutants in monocyte-derived macrophages and average levels of cumulative RT production by macrophages infected with the NL4-3 variants over a 20 day period. Values give averages +/- SEM of macrophages from three different donors with two independent virus stocks containing 1 ng p24 antigen. PSL, photon-stimulated luminescence. 


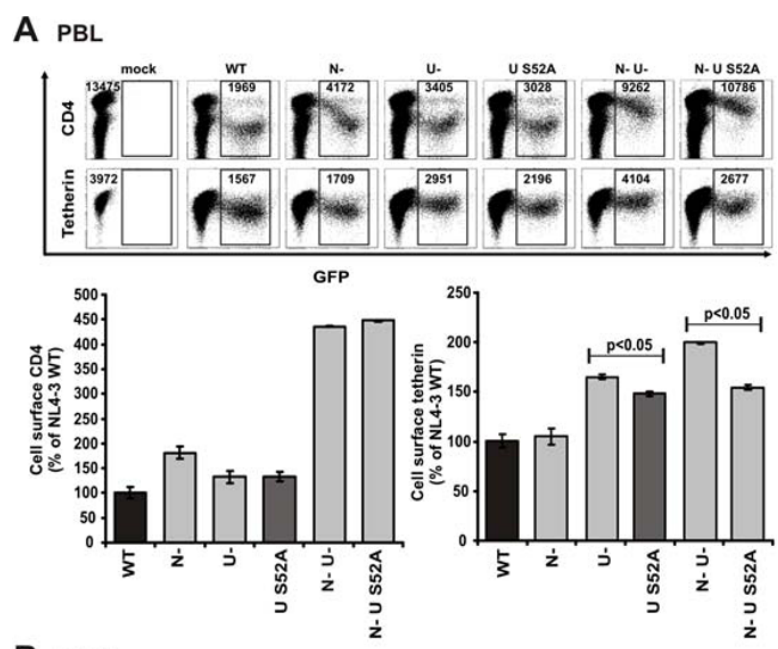

B MDM


Figure 6 Modulation of tetherin and CD4 in primary T-cells and macrophages by Vpu. (A) FACS analysis of CD4 and tetherin modulation in infected PBL cultures. PBL were infected with HIV-1 variants expressing eGFP via an IRES. Cells were stained with antibodies and measured by flow cytometry three days later. To quantify modulation of cell surface expressed CD4 and tetherin MFI of PBLs infected with HIV-1 NL4-3 WT was set as 100\%. Depicted are means +/- SD derived from experiments with four different donors. (B) Primary macrophages were infected with the indicated R5-tropic virus stocks expressing eGFP via an IRES. Cells were analyzed for cell surface MHC-I, CD4 and tetherin five days post infection similar to the PBL cultures. Presented are means +/- SD from infections with macrophages from three different donors each of those were infected with two independent virus stocks.

lower than in cells infected with HIV-1 containing an entirely defective $v p u$ gene (Fig. 6A, right). Thus, Vpu S52A down-modulates tetherin from HIV-1 infected $\mathrm{T}$-cells, albeit with lower efficiency than wildtype Vpu.

Next, we assessed if our viruses allow us to investigate the modulation of cell surface expressed receptors in macrophages, and we measured the down-modulation of MHC-I as a control. Inactivation of Nef resulted in about 2.5 fold higher MHC-I surface levels compared to WT infected MDM (Fig. 6B). Surprisingly, CD4 expression levels in HIV-1 infected MDM were comparably to uninfected cells, irrespective of Vpu or Nef expression (Fig 6B, left). Moreover, Vpu as well as the S52A mutant had similar minor effects on the levels of cell surface tetherin in MDM (Fig. 6B, right). Notably, MDMs infected with Nef-defective HIV-1 expressed lower levels 
of tetherin (Fig. 6B, right). Thus, Nef seems to induce tetherin cell surface expression in HIV-1 infected macrophages, perhaps as a result of Nef induced release of inflammatory cytokines [35].

In summary, our experiments demonstrate that VpuS52A reduces the levels of cell surface expressed tetherin in PBL, whereas it does not in macrophages.

\section{Discussion}

In the present study we demonstrate that the S52A mutation in Vpu impairs the ability of HIV-1 to replicate in macrophages, but not in ex vivo infected HLT cultures or PBL. This difference is most likely due to a reduced capability in counteracting tetherin, as the S52A Vpu mutant virus showed a wildtype phenotype in cells that express relatively low levels of this restriction factor, i.e. P4-CCR5 and T-cells, and a vpu-defective phenotype in cells that express higher levels, such as macrophages, 293T and T-cells transiently transfected with relatively high amounts of tetherin expression plasmids.

These data explain why it has been controversial whether the CK-II phosphorylation site in $\mathrm{Vpu}$ is only critical for CD4 degradation or is also relevant for virion release [8,12-15]. Indeed, we and others have found that $\mathrm{S} 52$ in Vpu is involved in the down-modulation and the degradation of tetherin (Fig. 1, 6) [7,9-11,19]. While most groups investigated $\mathrm{Vpu}$ with mutations in both serines at positions 52 and 56 (S2/6), we utilized the Vpu S52A mutant in our experiments. In the 293T experiments, S52A showed a similar phenotype like S2/6 (Fig. 1A-D and data not shown), which is in agreement with a recent report that also utilized the S52A variant [11]. This suggests that mutation of S52 alone is sufficient to disrupt the CK-II phosphorylation site in Vpu. Furthermore, we establish that phosphorylation by CKII is clearly important for Vpu to degrade tetherin by the use of three different CK-II inhibitors (Fig. 1E).

One possible explanation of the remaining antitetherin activity of the S52A mutant is that Vpu uses alternative pathways to counteract the restriction factor. On the other hand, Vpu containing mutations at the serine residues at position 52 and 56 has been shown to be able to bind to tetherin [10]. This could explain why the S52A Vpu exerts some residual counteracting activity, despite the fact that it does not efficiently induce tetherin degradation.

More importantly, our data suggest that the ability of $\mathrm{Vpu}$ to counteract tetherin is particularly required for HIV-1 replication in macrophages which are involved in virus transmission, the establishment of viral reservoirs, and neurological disorders associated with HIV-1 infection [36-38]. Thus, a reduced capability of Vpu to antagonize tetherin and to promote the release of progeny virions from macrophages may have important consequences for
HIV-1 transmission and pathogenicity. This is also highlighted by a recent report, demonstrating that only pandemic HIV-1 M expresses a fully functional Vpu protein, whereas the rarely distributed HIV- $1 \mathrm{~N}$ and $\mathrm{O}$ groups contain Vpu proteins that either are impaired in CD4 or tetherin degradation [39]. Conversely, it is remarkable that HIV-1 expressing a Vpu protein which is severely impaired in its ability to counteract tetherin, replicates efficiently in PBL and HLT and depletes CD4+ T-cells, particularly since $\mathrm{Vpu}$ is considered as a target for antiviral therapy [40]. Thus, Vpu inhibitors might need to be combined with agents inducing tetherin to achieve significant beneficial effects. In vitro this can be achieved by treatment of human cells with interferon-alpha $[6,20]$. Interestingly, interferon-alpha is upregulated by HIV-1 infection $[41,42]$ which may subsequently lead to the induction of tetherin in a feedback mechanism. Indeed we observed strong attenuation of viral replication in HLT and PBL in the presence of $100 \mathrm{U} / \mathrm{ml}$ interferon-alpha, irrespective of an intact $v p u$ gene (data not shown). This is in line with other reports [38-40] and could be explained by the fact that a variety of genes are upregulated in response to interferon-alpha, and additional pathways are triggered that might interfere with HIV-1 production [40-43].

Interestingly, among the predominant HIV-1 target cells in vivo, tetherin is highly expressed on macrophages (this study, [20]) and dendritic cells [43,44]. Thus, the ability of HIV-1 to efficiently counteract tetherin might have an impact on the cellular tropism of the virus. Both cell types become HIV-1 infected by the usage of the CCR5 co-receptor. Thus, it is also tempting to speculate that viral co-receptor tropism, i.e. the usage of CCR5 for viral entry segregates with the ability of $\mathrm{Vpu}$ to efficiently counteract tetherin. As already mentioned above, tetherin might be induced during HIV-1 infection by interferon-alpha, whose serum levels correlate with disease progression [45-47]. Therefore, our data carefully raise the possibility that the emergence of CXCR4 using HIV-1 variants during infection [48], might at least in part be also driven by increased expression of tetherin on the target cells. Currently it is not known whether primary HIV-1 $v p u$ alleles differ in their ability to counteract tetherin. To challenge these hypotheses, studies investigating the anti-tetherin activity of HIV-1 $v p u$ alleles from viruses isolated during different stages of infection and with different co-receptor tropism are warranted.

\section{Methods}

\section{Plasmids and proviral constructs}

For functional analysis, we generated vectors co-expressing Vpu or VpuS52A and GFP from a single bicistronic RNA via an internal ribosome entry site (IRES), as initially described for the analysis of Nef function [49]. 
Briefly HIV-1 NL4-3 Vpu was amplified with primers introducing unique $\mathrm{XbaI}$ and MluI restriction sites and subcloned into the pCGCG-IRES-GFP vector [50]. AU1-tagged Vpu and VpuS52A variants were constructed by introducing the DTYRYI-sequence at the $\mathrm{C}$-terminus together with the MluI primer. Site directed mutagenesis was utilized to introduce the S52A change in NL4-3 Vpu. The HIV-1 NL4-3 proviral constructs carrying disrupting mutations in $n e f, v p u$ or both viral genes have been previously described [27]. Splice overlap extension PCR was used to introduce mutation S52A in HIV-1 NL4-3 vpu and the element was subcloned by using the unique restriction sites StuI in env and the PflmI site just downstream of the pol gene, respectively. R5-tropic HIV-1 NL4-3 variants were constructed by exchanging the V3-loop region of NL4-3 with the one from the R5-tropic 92th014.12 isolate [51] by using the unique restriction sites StuI and NheI. HIV-1 NL4-3 variants co-expressing eGFP via an IRES were constructed by subcloning of fragments containing mutations in nef or $v p u$ in the pBR-NL4-3-IRES eGFP backbone $[25,34]$. The pECFP-tetherin construct was cloned by amplification of tetherin from a cDNA library (Spring Bioscience) introducing the single cutter restriction sites XhoI and EcoRI. The fragment was cloned in the pECFP-C1 vector backbone (Clontech). An untagged tetherin plasmid was cloned by amplification of tetherin with primers introducing $X b a I$ and $M l u I$ sites and subcloning in the pCGCG vector [50]. The IRES-GFP cassette was removed by digestion and religation with BamHI. The integrity of all PCR-derived inserts was verified by sequence analysis.

\section{Cell culture, transfections, virus stocks, p24 release and} infectivity assays

P4-CCR5, 293T and Jurkat cells were cultured as described previously [25,50]. P4-CCR5 and 293T cells were maintained in Dulbecco's modified Eagle's medium containing $10 \%$ heat-inactivated fetal bovine serum. The human Jurkat T-cell line was cultured in RPMI1640 medium supplemented with $10 \%$ fetal calf serum and antibiotics. PBMC were generated by Ficoll gradient centrifugation [34] and PBLs were recovered post plastic adherence of monocytes. To generate primary macrophage cultures PBMCs from healthy human donors were isolated using lymphocyte separation medium and macrophages were generated in teflon tubes (CellGenix) and cultured as described before [52,53]. Transfection of Jurkat T-cells was performed using the DMRIE-C reagent (Invitrogen, Gibco) following manufacturer's instructions. Furthermore, electroporation of Jurkat T-cells with proviral constructs and tetherin expression plasmids was performed with the MP-100 microporator device (PeqLab) as recommended by the manufacturer. Briefly, $4 \mu \mathrm{g}$ of proviral constructs co-expressing GFP were electroporated with the indicated amounts of tetherin plasmid. Two days post infection GFP+ cells were determined by FACS and the amount of released p24 was quantified in the supernatants using a p24 ELISA provided by the "AIDS \& Cancer virus program" (NCI, Frederick). P4-CCR5-cells were transfected using magnetic assisted transfection (IBA Tagnology) following standard protocols of the manufacturer. Briefly, 4000 P4-CCR5 cells per well were sown into 96-well plates one day prior to transfection. For transfection 0.1 $\mu \mathrm{g}$ of proviral DNA was co-incubated with $0.1 \mu \mathrm{l}$ MaTRA-A reagent in $15 \mu$ l OMEM (optimized minimum essential media, GIBCO) per well for $30 \mathrm{~min}$. Three days post transfection supernatants were harvested and analyzed for $\mathrm{p} 24$ antigen concentrations and $\beta$-galactosidase activity. To generate viral stocks, 293T cells were transfected with the proviral NL4-3 constructs by the calcium chloride method as already described [25,34]. Virus stocks and supernatants of transfected or infected cells to assess p24 release were quantified using the p24 ELISA described above. Virus infectivity was determined using P4-CCR5 cells as described [25]. Briefly, 4000 cells per well were sown out in 96-well-dishes in a volume of $100 \mu \mathrm{l}$ and infected after overnight incubation with virus stocks containing $1 \mathrm{ng}$ of p24 antigen. Three days post-infection viral infectivity was detected using the Gal screen kit from TROPIX as recommended by the manufacturer. $\beta$-galactosidase activities were detected as relative light units per second (RLU/s) in a microplate reader.

\section{Flow cytometric analysis}

CD4 and GFP reporter expression levels in Jurkat cells coexpressing Vpu and eGFP were measured as described previously for the analysis of Nef function [50]. Retention of newly synthesized CD4 from the endoplasmic reticulum to the cell surface in $293 \mathrm{~T}$ cells was measured by standard calcium chloride co-transfection of $1 \mu \mathrm{g}$ pCDNA-CD4 plasmid with $4 \mu \mathrm{g}$ pCG plasmid expressing Vpu, VpuS52A or GFP only. Cells were harvested and stained for FACS analysis 2 days post transfection essentially as described previously [50]. pECFP-tetherin and GFP expression in $293 \mathrm{~T}$ cells were analyzed similar to CD4 expression, but on a FACSAria equipped with a $405 \mathrm{~nm}$ laser. For the CKII inhibition experiments, we used Tyrphostin AG1112 (Sigma), Cay10577 (Biozol) and DRB (EnzoLife) reconstituted in DMSO. The concentrations used did not induce cytotoxic effects as determined by FACS FSC/SSC and MTT test (data not shown). PBLs and MDMs were infected with VSVG pseudotyped virus stocks containing $50 \mathrm{ng}$ p24. PBLs were analyzed by flow cytometry three days post infection for $\mathrm{CD} 4$ and tetherin expression as already described [34]. Similarly, primary macrophage cultures were trypsinized five days post infection and stained for CD4 and MHC-I expression as before [54]. Cell surface 
tetherin was measured by staining of PBLs or MDMs with 1:50 dilutions of the anti-tetherin/CD317 HM1.24 mAb kindly provided by Chugai Pharmaceuticals. As secondary antibody we used an 1:100 diluted Alexa633-conjugated goat anti-mouse antibody (Invitrogen).

\section{Immunoblotting}

$293 \mathrm{~T}$ cells were transfected with $5 \mu \mathrm{g}$ of vector DNA as described above. Two days post-transfection cells were pelleted, lysates were generated and separated through 12\% SDS-PAGE. Expression of Vpu in whole cellular lysates was analyzed by immunoblot using 1:500 diluted mouse anti-AU1 AB (Covance) or 1:500 dilutions of rabbit anti-Vpu sera kindly provided by U.Schubert [55]. GFP was detected with a 1:2000 dilution of rabbit antiGFP AB (Abcam). Viral envelope, GAG and Nef was visualized by 1:5000 dilutions of human-anti-HIV-1 gp120 Ab2G12, 1:5000 diluted rabbit-anti-HIV-1 p24 (provided by the AIDS \& Cancer virus program) and 1:1000 dilutions of mouse-anti-HIV-1 Nef (aa151-170) (ABI). For WB-analyses of cellular tetherin we lysed $293 \mathrm{~T}$ cells that were transfected with different amounts of tetherin as well as P4-CCR5, PBMC, PBL and MDMs and quantified total protein content using the 2D-quant kit (Amersham Biosciences). $40 \mu \mathrm{g}$ protein of each lysate were separated on a $15 \%$-SDS-bisacrylamide gel. For analysis of tetherin we used mouse anti-tetherin (B01P, Abnova) at a concentration of 1:500 and secondary goat anti-mouse (Jackson Immuno Research) at 1:10000 for ECL detection. To analyze enhancement of p24 release in the presence of tetherin we transfected $400.000293 \mathrm{~T}$ cells with proviral constructs $(5 \mu \mathrm{g})$ and different amounts of tetherin (10, 20 and $100 \mathrm{ng})$. Two days post infection, we pelleted the filtered supernatants and lysed them as well as the producer cells with 50-100 $\mu$ l RIPA buffer. Western blots were performed as described above, and band intensity was quantified using the Gene-snap software.

\section{Ex vivo-infected HLT}

HIV-1 replication and cytopathicity in ex vivo-infected HLTwas determined as described previously [26,27]. Briefly, human tonsillar tissue removed during routine tonsillectomy was received within 5 hours of excision. The tonsils were washed thoroughly with medium containing antibiotics and sectioned into 2- to $3-\mathrm{mm}^{3}$ blocks. These tissue blocks were placed on top of collagen sponge gels and infected with virus stocks containing $0.5 \mathrm{ng}$ p24 antigen essentially as described previously [26,27]. Supernatants were collected at three day intervals and productive HIV-1 infection was assessed by measuring p24 antigen content. Flow cytometry was performed on cells mechanically isolated from control and infected tissue blocks and depletion of CD4+ T-cells was quantified as described previously $[26,27]$. For determination of the CD4+/CD8+-Tcell ratio, cells were stained for surface markers by using
anti-CD3 fluorescein isothiocyanate (FITC), anti-CD4allophycocyanin (APC), and anti-CD8 Tri color.

Viral replication in primary macrophage and PBL cultures Human monocyte-derived macrophages (MDM) were isolated as described in "cell culture, virus stocks and transfection" and infected with $1 \mathrm{ng}$ p24 of R5-tropic HIV-1 NL4-3 variants. To assess viral spread and replication, aliquots of the infected cell culture supernatants were taken in two to three day intervals and stored at $-20^{\circ} \mathrm{C}$. Viral replication was determined by RT-assay essentially as described previously [25]. Similarly, PBLs were infected with $1 \mathrm{ng}$ p24 of X4-tropic HIV-1 NL4-3 variants expressing GFP via an IRES. For measurement of viral spread and transmission we took aliquots of infected cells in two or three days intervals and determined the amount of GFP+ cells by FACS as before [25].

\section{Statistical analysis}

All statistical calculations were performed with a oneway analysis of variances (ANOVA) using Graph Pad Prism Version 5.0. Correlations were calculated with the linear regression module.

\footnotetext{
Additional file 1: Supplementary Figure S1. Assessment of viral release by quantitative WB correlates with p24 ELISA. Correlation of the quantitative WB data shown in Fig. 2 with ELISA results, that were measured before the supernatants were pelleted. Click here for file

[http://www.biomedcentral.com/content/supplementary/1742-4690-7-1S1.PDF ]

Additional file 2: Supplementary Figure S2. HeLa derived P4-CCR5 cells express low levels of tetherin. Western blot analysis of endogenous tetherin expression in primary cells and HeLa-derived P4CCR5 cells. PBMC were either left untreated or stimulated with $1 \mu \mathrm{g} / \mathrm{ml}$ PHA for 24 hours (PBMC+)

Click here for file

[http://www.biomedcentral.com/content/supplementary/1742-4690-7-1S2.PDF ]

Additional file 3: Supplementary Figure S3. Vpu S52A is dispensable for HIV-1 release in primary blood lymphocytes (PBL) and Jurkat T-cells. (A) Replication kinetic of the indicated X4-tropic HIV1 isolates expressing eGFP via an IRES in PBL cultures. PBLs were infected with $1 \mathrm{ng}$ p24 and analyzed for the amount of GFP+ cells in two or three days intervals. Means +/- SD are calculated from infections of PBLs from two donors with three independent virus stocks. (B) The ability of Vpu S52A to enhance HIV-1 release from Jurkat T-cells is inhibited in a tetherin-dependent manner. $1{ }^{*} 10 \wedge 6$ Jurkat cells were electroporated with the different proviral constructs coexpressing GFP and the indicated amount of tetherin expression plasmid as described in the methods section. Two days post electroporation the percentage of GFP+ cells as well as p24 contents of the supernatants were quantified. Presented are means and standard deviations from triplicate electroporations from one representative out of three independent experiments.

Click here for file

[http://www.biomedcentral.com/content/supplementary/1742-4690-7-1S3.PDF ]
}

\section{Acknowledgements}

The authors thank Thomas Mertens, Heinrich Hohenberg and Volker Uhl for support, Kerstin Regensburger, Daniela Krnavek and Martha Mayer for technical assistance, Gerhard Rettinger, Herbert Riechelmann, Tilman Keck and Kai-Johannes Lorenz for providing tonsils, Chugai Pharmaceuticals for 
the HM1.24 mAb and Ulrich Schubert for providing the rabbit anti-Vpu serum. This work was supported by the Wilhelm-Sander Stiftung, NIH grant R01Al067057 the Deutsche Forschungsgemeinschaft and the Stiftung für neurovirale Erkrankungen. PW is supported by a fellowship from the Studienstiftung des Deutschen Volkes.

\section{Author details}

${ }^{1}$ Heinrich-Pette-Institute for Experimental Virology and Immunology, Martinistrasse 52, 20251 Hamburg, Germany. ${ }^{2}$ Institute of Virology, University of Ulm, Albert-Einstein-Allee 11, 89081 Ulm, Germany. ${ }^{3}$ Current address: Emory University, Atlanta GA 30322, USA.

\section{Authors' contributions}

Conceived and designed the experiments: MS FK. Performed the experiments: MS DR CB PW HK AI AS DS. Analyzed the data: MS DR CB PW FK. Contributed reagents/materials/analysis tools: MS TD FK. Wrote the paper: MS

\section{Competing interests}

The authors declare that they have no competing interests.

\section{Received: 31 August 2009}

Accepted: 15 January 2010 Published: 15 January 2010

\section{References}

1. Binette J, Cohen EA: Recent advances in the understanding of HIV-1 Vpu accessory protein functions. Curr Drug Targets Immune Endocr Metabol Disord 2004, 4:297-307.

2. Levesque $K$, Zhao YS, Cohen EA: Vpu exerts a positive effect on HIV-1 infectivity by down-modulating CD4 receptor molecules at the surface of HIV-1-producing cells. J Biol Chem 2003, 278:28346-28353.

3. Willey RL, Maldarelli F, Martin MA, Strebel K: Human immunodeficiency virus type 1 Vpu protein induces rapid degradation of CD4. J Virol 1992, 66:7193-7200.

4. Binette J, Dube M, Mercier J, Halawani D, Latterich M, Cohen EA: Requirements for the selective degradation of CD4 receptor molecules by the human immunodeficiency virus type $1 \mathrm{Vpu}$ protein in the endoplasmic reticulum. Retrovirology 2007, 4:75.

5. Neil SJ, Sandrin V, Sundquist WI, Bieniasz PD: An interferon-alpha-induced tethering mechanism inhibits HIV-1 and Ebola virus particle release but is counteracted by the HIV-1 Vpu protein. Cell Host Microbe 2007, 2:193-203.

6. Neil SJ, Zang T, Bieniasz PD: Tetherin inhibits retrovirus release and is antagonized by HIV-1 Vpu. Nature 2008, 451:425-30.

7. Van Damme N, Goff D, Katsura C, Jorgenson RL, Mitchell R, Johnson MC, Stephens EB, Guatelli J: The interferon-induced protein BST-2 restricts HIV-1 release and is downregulated from the cell surface by the viral Vpu protein. Cell Host Microbe 2008, 3:245-252.

8. Margottin F, Bour SP, Durand H, Selig L, Benichou S, Richard V, Thomas D, Strebel K, Benarous R: A novel human WD protein, h-beta $\operatorname{Tr} C p$, that interacts with HIV-1 Vpu connects CD4 to the ER degradation pathway through an F-box motif. Mol Cell 1998, 1:565-574.

9. Mitchell RS, Katsura C, Skasko MA, Fitzpatrick K, Lau D, Ruiz A, Stephens EB, Margottin-Goguet F, Benarous R, Guatelli JC: Vpu antagonizes BST-2mediated restriction of HIV-1 release via beta-TrCP and endo-lysosomal trafficking. PLOS Pathog 2009, 5:e1000450.

10. Douglas JL, Viswanathan K, McCarroll MN, Gustin JK, Fruh K, Moses AV: Vpu Directs the Degradation of the HIV Restriction Factor BST-2/tetherin via a \{beta\}TrCP-dependent Mechanism. J Virol 2009, 83(16):7931-47.

11. Mangeat B, Gers-Huber G, Lehmann M, Zufferey M, Luban J, Piguet V: HIV-1 Vpu neutralizes the antiviral factor Tetherin/BST-2 by binding it and directing its beta-TrCP2-dependent degradation. PLoS Pathog 2009, 5 e1000574

12. Schubert $U$, Henklein $P$, Boldyreff B, Wingender $E$, Strebel $K$, Porstmann $T$ : The human immunodeficiency virus type 1 encoded Vpu protein is phosphorylated by casein kinase-2 (CK-2) at positions Ser52 and Ser56 within a predicted alpha-helix-turn-alpha-helix-motif. J Mol Biol 1994, 236:16-25.

13. Schubert U, Strebel K: Differential activities of the human immunodeficiency virus type 1-encoded Vpu protein are regulated by phosphorylation and occur in different cellular compartments. J Virol 1994, 68:2260-2271.

14. Friborg J, Ladha A, Gottlinger H, Haseltine WA, Cohen EA: Functional analysis of the phosphorylation sites on the human immunodeficiency virus type 1 Vpu protein. J Acquir Immune Defic Syndr Hum Retrovirol 1995, 8:10-22.

15. Schubert U, Bour S, Ferrer-Montiel AV, Montal M, Maldarell F, Strebel K: The two biological activities of human immunodeficiency virus type $1 \mathrm{Vpu}$ protein involve two separable structural domains. J Virol 1996, 70:809-819.

16. Varthakavi V, Smith RM, Bour SP, Strebel K, Spearman P: Viral protein U counteracts a human host cell restriction that inhibits HIV-1 particle production. Proc Natl Acad Sci USA 2003, 100:15154-15159.

17. Sato K, Yamamoto SP, Misawa N, Yoshida T, Miyazawa T, Koyanagi Y: Comparative study on the effect of human BST-2/Tetherin on HIV-1 release in cells of various species. Retrovirology 2009, 6:53.

18. Bartee E, McCormack A, Fruh K: Quantitative membrane proteomics reveals new cellular targets of viral immune modulators. PLoS Pathog 2006, 2:e107.

19. Goffinet C, Allespach I, Homann S, Tervo HM, Habermann A, Rupp D, Oberbremer L, Kern C, Tibroni N, Welsch S, Krijnse-Locker J, Banting G, Kräusslich HG, Fackler OT, Keppler OT: HIV-1 antagonism of CD317 is species specific and involves Vpu-mediated proteasomal degradation of the restriction factor. Cell Host Microbe 2009, 5:285-297.

20. Miyagi E, Andrew AJ, Kao S, Strebel K: Vpu enhances HIV-1 virus release in the absence of Bst-2 cell surface down-modulation and intracellular depletion. Proc Natl Acad Sci USA 2009, 106(8):2868-73.

21. Wildum S, Schindler M, Munch J, Kirchhoff F: Contribution of Vpu, Env, and Nef to CD4 down-modulation and resistance of human immunodeficiency virus type 1-infected T cells to superinfection. J Virol 2006, 80:8047-8059.

22. Chen BK, Gandhi RT, Baltimore D: CD4 down-modulation during infection of human $T$ cells with human immunodeficiency virus type 1 involves independent activities of vpu, env, and nef. J Virol 1996, 70:6044-6053.

23. Ross TM, Oran AE, Cullen BR: Inhibition of HIV-1 progeny virion release by cell-surface CD4 is relieved by expression of the viral Nef protein. Curr Biol 1999, 9:613-621.

24. Munch J, Schindler M, Wildum S, Rucker E, Bailer N, Knoop V, Novembre FJ, Kirchhoff F: Primary sooty mangabey simian immunodeficiency virus and human immunodeficiency virus type 2 nef alleles modulate cell surface expression of various human receptors and enhance viral infectivity and replication. J Virol 2005, 79:10547-10560.

25. Munch J, Rajan D, Schindler M, Specht A, Rucker E, Novembre FJ, Nerrienet E, Muller-Trutwin MC, Peeters M, Hahn BH, Kirchhoff F: Nefmediated enhancement of virion infectivity and stimulation of viral replication are fundamental properties of primate lentiviruses. J Virol 2007, 81:13852-13864.

26. Glushakova S, Munch J, Carl S, Greenough TC, Sullivan JL, Margolis L, Kirchhoff F: CD4 down-modulation by human immunodeficiency virus type 1 Nef correlates with the efficiency of viral replication and with CD4(+) T-cell depletion in human lymphoid tissue ex vivo. J Virol 2001, 75:10113-10117.

27. Rucker E, Grivel JC, Munch J, Kirchhoff F, Margolis L: Vpr and Vpu are important for efficient human immunodeficiency virus type 1 replication and CD4+ T-cell depletion in human lymphoid tissue ex vivo. J Virol 2004, 78:12689-12693.

28. Glushakova S, Baibakov B, Margolis LB, Zimmerberg J: Infection of human tonsil histocultures: a model for HIV pathogenesis. Nat Med 1995, 1:1320-1322

29. Gondois-Rey F, Grivel JC, Biancotto A, Pion M, Vigne R, Margolis LB, Hirsch I: Segregation of R5 and X4 HIV-1 variants to memory T cell subsets differentially expressing CD62L in ex vivo infected human lymphoid tissue. AIDS 2002, 16:1245-1249.

30. Balliet JW, Kolson DL, Eiger G, Kim FM, McGann KA, Srinivasan A, Collman R: Distinct effects in primary macrophages and lymphocytes of the human immunodeficiency virus type 1 accessory genes vpr, vpu, and nef: mutational analysis of a primary HIV-1 isolate. Virology 1994, 200:623-631.

31. Swingler S, Mann A, Jacque J, Brichacek B, Sasseville VG, Williams K, Lackner AA, Janoff EN, Wang R, Fisher D, Stevenson M: HIV-1 Nef mediates lymphocyte chemotaxis and activation by infected macrophages. Nat Med 1999, 5:997-103. 
32. Richards KH, Clapham PR: Effects of vpu start-codon mutations on human immunodeficiency virus type 1 replication in macrophages. J Gen Virol 2007, 88:2780-2792.

33. Schubert U, Clouse KA, Strebel K: Augmentation of virus secretion by the human immunodeficiency virus type $1 \mathrm{Vpu}$ protein is cell type independent and occurs in cultured human primary macrophages and lymphocytes. J Virol 1995, 69:7699-7711.

34. Schindler M, Munch J, Kutsch O, Li H, Santiago ML, Bibollet-Ruche F, MullerTrutwin MC, Novembre FJ, Peeters M, Courgnaud V, Bailes E, Roques P, Sodora DL, Silvestri G, Sharp PM, Hahn BH, Kirchhoff F: Nef-mediated suppression of $\mathrm{T}$ cell activation was lost in a lentiviral lineage that gave rise to HIV-1. Cell 2006, 125:1055-1067.

35. Olivetta E, Percario Z, Fiorucci G, Mattia G, Schiavoni I, Dennis C, Jager J, Harris M, Romeo G, Affabris E, Federico M: HIV-1 Nef induces the release of inflammatory factors from human monocyte/macrophages: involvement of Nef endocytotic signals and NF-kappa B activation. $J$ Immunol 2003, 170:1716-1727.

36. Montaner LJ, Crowe SM, Aquaro S, Perno CF, Stevenson M, Collman RG: Advances in macrophage and dendritic cell biology in HIV-1 infection stress key understudied areas in infection, pathogenesis, and analysis of viral reservoirs. J Leukoc Biol 2006, 80:961-964.

37. Sharova N, Swingler C, Sharkey M, Stevenson M: Macrophages archive HIV1 virions for dissemination in trans. EMBO J 2005, 24:2481-2489.

38. Stevenson M: HIV-1 pathogenesis. Nat Med 2003, 9:853-860.

39. Sauter D, Schindler M, Specht A, Landford WN, Munch J, Kim KA, Votteler J, Schubert U, Bibollet-Ruche F, Keele BF, Takehisa J, Ogando Y, Ochsenbauer C, Kappes JC, Ayouba A, Peeters M, Learn GH, Shaw G, Sharp PM, Bieniasz P, Hahn BH, Hatziioannou T, Kirchhoff F: Tetherin-driven adaptation of $\mathrm{Vpu}$ and Nef function and the evolution of pandemic and nonpandemic HIV-1 strains. Cell Host Microbe 2009, 6:409-421.

40. Montal M: Vpu matchmakers as a therapeutic strategy for HIV infection. PLoS Pathog 2009, 5:e1000246.

41. Corbeil J, Sheeter D, Genini D, Rought $S$, Leoni L, Du P, Ferguson M, Masys DR, Welsh JB, Fink JL, Sasik R, Huang D, Drenkow J, Richman DD, Gingeras T: Temporal gene regulation during HIV-1 infection of human CD4+ T cells. Genome Res 2001, 11:1198-1204

42. Imbeault M, Ouellet M, Tremblay MJ: Microarray study reveals that HIV-1 induces rapid type-I interferon-dependent p53 mRNA up-regulation in human primary CD4+ T cells. Retrovirology 2009, 6:5.

43. Lehmann C, Harper JM, Taubert D, Hartmann P, Fatkenheuer G, Jung N, van Lunzen J, Stellbrink HJ, Gallo RC, Romerio F: Increased interferon alpha expression in circulating plasmacytoid dendritic cells of HIV-1-infected patients. J Acquir Immune Defic Syndr 2008, 48:522-530.

44. Blasius AL, Giurisato E, Cella M, Schreiber RD, Shaw AS, Colonna M: Bone marrow stromal cell antigen 2 is a specific marker of type I IFNproducing cells in the naive mouse, but a promiscuous cell surface antigen following IFN stimulation. J Immunol 2006, 177:3260-3265.

45. Lehmann C, Taubert D, Jung N, Fatkenheuer G, van Lunzen J, Hartmann P, Romerio F: Preferential upregulation of interferon-alpha subtype 2 expression in HIV-1 patients. AIDS Res Hum Retroviruses 2009, 25:577-581.

46. Calabrese LH, Proffitt MR, Gupta MK, Easley KA, Walker JR, Rehm SJ, Valenzuela R, Clough JD: Serum beta 2-microglobulin and interferon in homosexual males: relationship to clinical findings and serologic status to the human T lymphotropic virus (HTLV-III). AIDS Res 1984, 1:423-438.

47. Krown SE, Niedzwiecki D, Bhalla RB, Flomenberg N, Bundow D, Chapman D: Relationship and prognostic value of endogenous interferon-alpha, beta 2-microglobulin, and neopterin serum levels in patients with Kaposi sarcoma and AIDS. J Acquir Immune Defic Syndr 1991, 4:871-880.

48. Philpott SM: HIV-1 coreceptor usage, transmission, and disease progression. Curr HIV Res 2003, 1:217-227.

49. Greenberg ME, lafrate AJ, Skowronski J: The SH3 domain-binding surface and an acidic motif in HIV-1 Nef regulate trafficking of class I MHC complexes. EMBO J 1998, 17:2777-2789.

50. Schindler M, Wurfl S, Benaroch P, Greenough TC, Daniels R, Easterbrook P, Brenner M, Munch J, Kirchhoff F: Down-modulation of mature major histocompatibility complex class II and up-regulation of invariant chain cell surface expression are well-conserved functions of human and simian immunodeficiency virus nef alleles. J Virol 2003, 77:10548-10556.

51. Papkalla A, Munch J, Otto C, Kirchhoff F: Nef enhances human immunodeficiency virus type 1 infectivity and replication independently of viral coreceptor tropism. J Virol 2002, 76:8455-8459.
52. Andreesen R, Picht J, Lohr GW: Primary cultures of human blood-born macrophages grown on hydrophobic teflon membranes. J Immunol Methods 1983, 56:295-304.

53. von Briesen H, Andreesen R, Esser R, Brugger W, Meichsner C, Becker K, Rubsamen-Waigmann $\mathrm{H}$ : Infection of monocytes/macrophages by HIV in vitro. Res Virol 1990, 141:225-231.

54. Schindler M, Wildum S, Casartelli N, Doria M, Kirchhoff F: Nef alleles from children with non-progressive HIV-1 infection modulate MHC-II expression more efficiently than those from rapid progressors. AIDS 2007, 21:1103-1107.

55. Schubert U, Anton LC, Bacik I, Cox JH, Bour S, Bennink JR, Orlowski M, Strebel K, Yewdell JW: CD4 glycoprotein degradation induced by human immunodeficiency virus type $1 \mathrm{Vpu}$ protein requires the function of proteasomes and the ubiquitin-conjugating pathway. J Virol 1998, 72:2280-2288.

\section{doi:10.1186/1742-4690-7-1}

Cite this article as: Schindler et al:: Vpu serine 52 dependent counteraction of tetherin is required for HIV-1 replication in macrophages, but not in ex vivo human lymphoid tissue. Retrovirology $20107: 1$

\section{Publish with Biomed Central and every scientist can read your work free of charge}

"BioMed Central will be the most significant development for disseminating the results of biomedical research in our lifetime. "

Sir Paul Nurse, Cancer Research UK

Your research papers will be:

- available free of charge to the entire biomedical community

- peer reviewed and published immediately upon acceptance

- cited in PubMed and archived on PubMed Central

- yours - you keep the copyright
BioMedcentral 